\title{
Special Section Guest Editorial: Nanostructured Thin Films V: Fundamentals and Applications
}

\author{
Tom G. Mackay, ${ }^{a}$ Yi-Jun Jen, ${ }^{b}$ and Raúl J. Martín-Palma ${ }^{c}$ \\ ${ }^{a}$ School of Mathematics and Maxwell Institute for Mathematical Science, \\ University of Edinburgh, James Clerk Maxwell Building, King's Buildings, Edinburgh, \\ Scotland EH9 3JZ, United Kingdom, and Department of Engineering Science and Mechanics, \\ The Pennsylvania State University, University Park, PA, 16802, USA \\ t.mackay@ed.ac.uk \\ ${ }^{\mathrm{b}}$ Department of Electro-Optical Engineering, National Taipei University of Technology, \\ No. 1, Sec. 3, Chung-Hsiao E. Rd. Taipei, Taiwan (106) \\ jyjun@ntut.edu.tw \\ ${ }^{\mathrm{c}}$ Departamento de Física Aplicada, Universidad Autónoma de Madrid, Campus de Cantoblanco, \\ 28049 Madrid, Spain, and Department of Material Science and Engineering, \\ The Pennsylvania State University, University Park, PA, 16802, USA \\ rauljose.martin@uam.es
}

Nanotechnology has progressed by leaps and bounds since it became the focus of intense applied research activity in the late 20th century. In particular, thin films with engineered structures at nanometer length scales have figured prominently in this nanotechnological revolution. Their unique and sometimes exotic and counterintuitive properties present engineers and scientists with tantalizing opportunities and often demanding challenges. The list of potential applications for nanostructured thin films grows longer each year. This growth is matched, and often fueled, by the ever more complex nanostructures that can be achieved in practice due to advances in manufacturing techniques. The annual Nanostructured Thin Films conference, which is a constituent of the SPIE Optics + Photonics annual meeting, provides a forum for the exchange of the most recent developments in this rapidly developing field.

The fifth conference in the Nanostructured Thin Films series took place in San Diego (California, USA) between 14 and 16 August 2012. Around 50 works were submitted in total. The range of topics included nanostructured solar cells, fundamentals and applications, theoretical studies, biomimetic nanostructures, fabrication and characterization, optical detection, and sculptured thin films. The works were distributed over 10 sessions of oral presentations and a poster session. The presented research represented activity from all continents of the world.

This special section is composed of a set of selected papers that have been expanded from those presented at the Nanostructured Thin Films V conference. They provide a snapshot of the diverse range of research activities ongoing in this field internationally and bode well for future developments pertaining to nanostructured thin films.

\section{Acknowledgments}

The guest editors thank their program committee, all authors for their contributions to this special section, and all those who participated in the Nanostructured Thin Films V conference and contributed to its proceedings. 